\title{
LARGE DEVIATIONS OF EMPIRICAL MEASURES UNDER SYMMETRIC INTERACTION
}

\author{
WŁODZIMIERZ BRYC
}

\begin{abstract}
We prove the large deviation principle for the joint empirical measure of pairs of random variables which are coupled by a "totally symmetric" interaction. The rate function is given by an explicit bilinear expression, which is finite only on product measures and hence is non-convex.
\end{abstract}

\section{INTRODUCTION}

1.1. Large deviations of empirical measures have been widely studied in the literature since the celebrated Sanov's theorem, which gives the large deviations principle in the scale of $n$ of the empirical measures of i. i. d. random variables with the relative entropy $H(\mu \mid \nu)=\int \log \frac{d \mu}{d \nu} d \mu$ as the rate function. Another entropy, Voiculescu's non-commutative entropy $\Sigma(\mu)=\iint \log |x-y| \mu(d x) \mu(d y)$, arises in the study of fluctuations of eigenvalues of random matrices, see Hiai \& Petz ${ }^{8}$ and the references therein. Chan $\underline{4}$ interprets empirical measures of eigenvalues of random matrices as a system of interacting diffusions with singular interactions.

1.2. In this paper we study empirical measures which can be thought of as a decoupled version of the empirical measures generated by random matrices. We are interested in empirical measures on $\mathbb{R}^{2}$ generated by pairs of random variables that are tied together by a totally symmetric, and hence non-local, interaction, see formula (11) for the (unnormalized) joint density. Under certain assumptions, we prove that the large deviation principle in the scale $n^{2}$ holds for the joint empirical measures, and the rate function is non-convex. As a corollary, we derive a large deviations principle for the univariate average empirical measures with a rate function that superficially resembles the rate function of random matrices, see Corollary 1 an interesting feature here is the emergence of concave rate functions, see Remark 3. (Eigenvalues of random matrices are exchangeable and the large deviation rate function for their empirical measures is convex; infinite exchangeable sequences often lead to non-convex rate functions, see Dinwoodie \& Zabell(7) and [Ref. [3] Example 3].)

1.3. Let $g: \mathbb{R}^{2} \rightarrow \mathbb{R}$ be a continuous function which satisfies the following conditions.

Assumption 1. $g(x, y) \geq 0$ for all $x, y \in \mathbb{R}$.

Date: June 21, 2003.

2000 Mathematics Subject Classification. Primary: 60F10 Secondary: 60K35.

Key words and phrases. large deviations, symmetric interaction, non-convex rate function.

Department of Mathematics, University of Cincinnati, P.O. Box 210025, Cincinnati, OH 452210025. Email: Wlodzimierz.Bryc@UC.edu. 
Assumption 2. For every $0<\alpha \leq 1, M_{\alpha}:=\iint g^{\alpha}(x, y) d x d y<\infty$.

Assumption 3. $g(x, y)$ is bounded, $g(x, y) \leq e^{C}$.

In the following statements we use the convention that $-\log 0=\infty$.

Assumption 4. The function $k(x, y):=-\log g(x, y)$ has compact level sets: for every $a>0$ the set $\left\{(x, y): g(x, y) \geq e^{-a}\right\} \subset \mathbb{R}^{2}$ is compact.

The purpose of the next assumption is to allow singular interactions, where $g(x, x)=0$; this assumption is automatically satisfied with $\beta=0$ if $g(x, y)>0$ for all $x, y$.

Assumption 5. There is a $\beta \geq 0$ such that $(x, y) \mapsto \beta \log |x-y|-\log g(x, y)$ extends from $\{(x, y): x \neq y\}$ to the continuous function on $\mathbb{R}^{2}$.

Examples of functions that satisfy these assumptions are: the Gaussian kernel

$$
g(x, y)=e^{-x^{2}-y^{2}+2 \theta x y}
$$

for $|\theta|<1$, see the proof of Proposition 1 and a singular kernel

$$
g(x, y)=|x-y|^{\beta} e^{-x^{2}-y^{2}}
$$

for $\beta \geq 0$, see the proof of Proposition 2 .

Define

$$
f\left(x_{1}, \ldots, x_{n}, y_{1}, \ldots, y_{n}\right)=\prod_{i, j=1}^{n} g\left(x_{i}, y_{j}\right) .
$$

Clearly, $f$ depends on $n$; we will suppress this dependence in our notation and we will further write $f(\mathbf{x}, \mathbf{y})$ as a convenient shorthand for $f\left(x_{1}, \ldots, x_{n}, y_{1}, \ldots, y_{n}\right)$.

Assumptions 10 2. and 3 imply that $f$ is integrable. Indeed, since $g(x, y) \leq e^{C}$,

$$
\begin{gathered}
Z_{n}:=\int_{\mathbb{R}^{2 n}} f\left(x_{1}, \ldots, x_{n}, y_{1}, \ldots, y_{n}\right) d x_{1} \ldots d x_{n} d y_{1} \ldots d y_{n} \\
\leq \int_{\mathbb{R}^{2 n}} \prod_{i=1}^{n}\left(g\left(x_{i}, y_{i}\right) e^{C(n-1)}\right) d x_{1} \ldots d x_{n} d y_{1} \ldots d y_{n}=e^{C\left(n^{2}-n\right)} M_{1}^{n}<\infty .
\end{gathered}
$$

We are interested in joint empirical measures

$$
\hat{\mu}_{n}=\frac{1}{n^{2}} \sum_{i, j=1}^{n} \delta_{x_{i}, y_{j}}
$$

considered as random variables with values in the Polish space of probability measures $\mathcal{P}\left(\mathbb{R}^{2}\right)$ (equipped with the topology of weak convergence), with the distribution induced on $\mathcal{P}\left(\mathbb{R}^{2}\right)$ by the probability measure $\operatorname{Pr}=\operatorname{Pr}_{n} \in \mathcal{P}\left(\mathbb{R}^{2 n}\right)$ defined by

$$
\operatorname{Pr}(d \mathbf{x}, d \mathbf{y}):=\frac{1}{Z_{n}} f(\mathbf{x}, \mathbf{y}) d \mathbf{x} d \mathbf{y} .
$$

Theorem 1. If $g(x, y)$ satisfies Assumptions 1 , 20 8 and 5 , then the joint empirical measures $\left\{\hat{\mu}_{n}\right\}$ satisfy the large deviation principle in the scale $n^{2}$ with the rate function $\mathbb{I}: \mathcal{P}\left(\mathbb{R}^{2}\right) \rightarrow[0, \infty]$ given by

$$
\mathbb{I}(\mu)= \begin{cases}\iint k(x, y) \nu_{1}(d x) \nu_{2}(d y)-I_{0} & \text { if } \mu=\nu_{1} \otimes \nu_{2} \text { is a product } \\ & \text { measure and } k \text { is } \mu \text {-integrable } ; \\ \infty & \text { otherwise }\end{cases}
$$


where $k(x, y)=-\log g(x, y)$ and $I_{0}=\inf _{x, y \in \mathbb{R}} k(x, y)$.

Definition 1 ( [Ref. 2, Chapter 3]). We say that $k: \mathbb{R}^{2} \rightarrow \mathbb{R}$ is a negative definite kernel if $k(x, y)=k(y, x)$ and

$$
\sum k\left(x_{i}, x_{j}\right) c_{i} c_{j} \leq 0
$$

for all $x_{i}, c_{i} \in \mathbb{R}$ such that $\sum c_{i}=0$.

Condition (5) is satisfied for $k(x, y)=V(x)+W(y)-\kappa(x, y)$, where $\kappa(x, y)$ is positive-definite.

Consider the average empirical measures

$$
\hat{\sigma}_{n}:=\frac{1}{2 n} \sum_{i=1}^{n}\left(\delta_{x_{i}}+\delta_{y_{i}}\right) .
$$

Corollary 1. Suppose that the assumptions of Theorem 1 hold true, and in addition $k(x, y)$ is continuous and negative-definite. Then the average empirical measures $\left\{\hat{\sigma}_{n}\right\}$ satisfy the large deviation principle in the scale $n^{2}$ with the rate function

$$
\mathbb{I}(\nu)=\iint k(x, y) \nu(d x) \nu(d y)-I_{0},
$$

and $I_{0}=\inf _{x} k(x, x)$.

Proof. This follows from the contraction principle. The mapping $\mu() \mapsto$ $\frac{1}{2} \int \mu(\cdot, d y)+\frac{1}{2} \int \mu(d x, \cdot)$ is continuous in the weak topology. The rate function is $\mathbb{I}(\nu)=\inf \left\{\mathbb{I}\left(\nu_{1} \otimes \nu_{2}\right): \nu=\frac{1}{2} \nu_{1}+\frac{1}{2} \nu_{2}\right\}$.

Write $\mathbb{K}(\mu)=\iint k(x, y) \mu(d x, d y)$. If $\nu=\frac{1}{2} \nu_{1}+\frac{1}{2} \nu_{2}$ then [Ref. 10, Theorem 3] implies that $\mathbb{K}\left(\nu_{1} \otimes \nu_{2}\right) \geq \mathbb{K}(\nu \otimes \nu)$. Thus $\mathbb{I}(\nu)=\mathbb{K}(\nu \otimes \nu)-I_{0}$.

Another form of the cited inequality is that for any two probability measures $\nu_{1}, \nu_{2}$ we have

$$
2 \mathbb{K}\left(\nu_{1} \otimes \nu_{2}\right) \geq \mathbb{K}\left(\nu_{1} \otimes \nu_{1}\right)+\mathbb{K}\left(\nu_{2} \otimes \nu_{2}\right) .
$$

In particular, $2 k(x, y) \geq k(x, x)+k(y, y)$, which implies that $I_{0}=\inf _{x, y} k(x, y)=$ $\inf _{x} k(x, x)$.

Remark 1. Inequality (6) implies that the rate function satisfies

$$
\mathbb{I}\left(\frac{1}{2} \nu_{1}+\frac{1}{2} \nu_{2}\right) \geq \frac{1}{2} \mathbb{I}\left(\nu_{1}\right)+\frac{1}{2} \mathbb{I}\left(\nu_{2}\right) .
$$

\section{Applications}

2.1. Let $g(x, y)=e^{-x^{2}-y^{2}+2 \theta x y}$. Then

$$
f(\mathbf{x}, \mathbf{y})=\exp \left(-n \sum_{i=1}^{n} x_{i}^{2}-n \sum_{j=1}^{n} y_{j}^{2}+2 \theta \sum_{i, j=1}^{n} x_{i} y_{j}\right)
$$

and $k(x, y)=x^{2}+y^{2}-2 \theta x y$.

Denote by $m_{r}(\nu)=\int x^{r} \nu(d x)$ the $r$-th moment of a measure $\nu$.

Proposition 1. (i) If $|\theta|<1$ then the empirical measures

$$
\hat{\nu}_{n}:=\frac{1}{n} \sum_{i=1}^{n} \delta_{x_{j}}
$$


satisfy the large deviation principle in the scale $n^{2}$ with the rate function

$$
\mathbb{I}(\nu)=\left(m_{2}(\nu)-\theta^{2} m_{1}^{2}(\nu)\right)
$$

(ii) If $0 \leq \theta<1$ then the average empirical measures

$$
\hat{\sigma}_{n}:=\frac{1}{2 n} \sum_{i=1}^{n}\left(\delta_{x_{i}}+\delta_{y_{i}}\right)
$$

satisfy the large deviation principle in the scale $n^{2}$ with the rate function

$$
\mathbb{I}(\nu)=2\left(m_{2}(\nu)-\theta m_{1}^{2}(\nu)\right) .
$$

(In the formulas above, use $\mathbb{I}(\nu)=\infty$ if $m_{2}(\nu)=\infty$.)

Remark 2. The marginal density relevant in Proposition 1 (i) is

$$
f_{1}(\mathbf{x})=C(n, \theta) \exp \left(-n^{2}\left(\frac{1}{n} \sum_{i=1}^{n} x_{i}^{2}-\theta^{2}\left(\frac{1}{n} \sum_{i=1}^{n} x_{i}\right)^{2}\right)\right) .
$$

Remark 3. Both rate functions in Proposition 1 are concave.

Proof. (i) It is easy to see that the assumptions of Theorem 1 are satisfied. Indeed, $k(x, y)=(x-\theta y)^{2}+\left(1-\theta^{2}\right) y^{2}$ is continuous, bounded from below. Furthermore

$$
\left\{k(x, y) \leq a^{2}\right\} \subset\left\{|y| \leq|a| /\left(1-\theta^{2}\right)\right\} \cap\left\{|x| \leq|a| /\left(1-\theta^{2}\right)\right\}
$$

so $k(x, y)$ has compact level sets. Finally, for $\alpha>0$ by a change of variables we see that $\iint e^{-\alpha k(x, y)} d x d y=\frac{1}{\alpha} \iint e^{-k(x, y)} d x d y<\infty$ so Assumption 2 is satisfied, too.

The result follows by the contraction principle: taking a marginal of a measure in $\mathcal{P}\left(\mathbb{R}^{2}\right)$ is a continuous mapping. The rate function is $\inf \{\mathbb{I}(\mu): \nu(A)=$ $\mu(A \times \mathbb{R})\}$. But since $\mathbb{I}$ is infinite on non-product measures, this is the same as $\inf _{\nu_{2}}\left\{\iint k(x, y) \nu(d x) \nu_{2}(d y)-I_{0}\right\}$. Since $I_{0}=0$ here, it remains to notice that $\inf _{\nu_{2}}\left\{\iint k(x, y) \nu(d x) \nu_{2}(d y)\right\}=\inf _{y}\left\{\int k(x, y) \nu(d x)\right\}=\inf _{y}\left\{m_{2}(\nu)+y^{2}-\right.$ $\left.2 \theta y m_{1}(\nu)\right\}=m_{2}(\nu)-\theta^{2} m_{1}^{2}(\nu)$.

(ii) This follows from Corollary 1 if $\theta \geq 0$ then $2 \theta x y$ is positive-definite. Thus $k(x, y)=x^{2}+y^{2}-2 \theta x y$ is a negative definite kernel.

2.2. Next, we consider a model which can be interpreted as a "decoupled" version of a model studied in relation to eigenvalue fluctuations of random matrices, where one encounters $x_{j}$ instead of our $y_{j}$, compare [Ref. 1] Section 5], [Ref. 9] formula (1.9)]. We consider here a slightly more general situation when

$$
g(x, y)=|x-y|^{\beta} e^{-V(x)-W(y)} .
$$

Then

$$
f(\mathbf{x}, \mathbf{y})=\prod_{i, j=1}^{n}\left|x_{i}-y_{j}\right|^{\beta} \prod_{i=1}^{n} e^{-n V\left(x_{i}\right)} \prod_{j=1}^{n} e^{-n W\left(y_{j}\right)},
$$

and $k(x, y)=V(x)+W(y)-\beta \log |x-y|$. We assume that functions $V(x), W(y)$ are continuous, $\beta \geq 0$, and that

$$
\lim _{|x| \rightarrow \infty} \frac{V(|x|)}{\log \sqrt{1+x^{2}}}=\lim _{|y| \rightarrow \infty} \frac{W(|y|)}{\log \sqrt{1+y^{2}}}=\infty .
$$


Proposition 2. The bivariate empirical measures $\hat{\mu}_{n}$ defined by (2) satisfy the large deviation principle in the scale $n^{2}$ with the rate function $\mathbb{I}$ given by (4).

In particular, if $V(u)=W(u)=u^{2}$, then the rate function is

$$
\mathbb{I}\left(\nu_{1} \otimes \nu_{2}\right)=m_{2}\left(\nu_{1}\right)+m_{2}\left(\nu_{2}\right)-\beta \iint \log |x-y| \nu_{1}(d x) \nu_{2}(d y)+1 / 2 \beta(\log \beta-1) .
$$

Proof. We verify that the hypotheses of Theorem 1 are satisfied. Assumption 1 holds trivially. Assumption 5 holds trivially since $V(x)+W(y)$ is continuous.

To verify Assumption 3 notice that

$$
k(x, y) \geq V(x)+W(y)-\beta \log \sqrt{1+x^{2}}-\beta \log \sqrt{1+y^{2}} .
$$

Since $V(x)-\beta \log \sqrt{1+x^{2}}$ is a continuous function which by (7) tends to infinity as $x \rightarrow \pm \infty$, it is bounded from below, $V(x)-\beta \log \sqrt{1+x^{2}} \geq-c$ for some $c$. Similarly, $W(y)-\beta \log \sqrt{1+y^{2}} \geq-c$.

We now verify Assumption 4 The set $K_{a}:=\{k(x, y) \leq a\}$ is closed since $k$ is lower semicontinuous. Furthermore, (8) implies that $K_{a}$ is contained in a level set of the continuous function $V(x)+W(y)-\beta \log \sqrt{1+x^{2}}-\beta \log \sqrt{1+y^{2}}$. The latter set is bounded since $V(x)-\beta \log \sqrt{1+x^{2}}>a+c$ for all large enough $|x|$ and similarly $W(y)-\beta \log \sqrt{1+y^{2}}>a+c$ for all large enough $|y|$.

To verify Assumption 2 we use inequality (8) again. It implies

$$
\iint g(x, y)^{\alpha} d x d y \leq \int e^{-\alpha\left(V(x)-\beta \log \sqrt{1+x^{2}}\right)} d x \int e^{-\alpha\left(W(y)-\beta \log \sqrt{1+y^{2}}\right)} d y .
$$

By assumption (7), there is $N>0$ such that for $|x|>N$ we have $V(x)>(\beta+2 / \alpha) \log \sqrt{1+x^{2}}$. By the previous argument the integrand is bounded; thus $\int e^{-\alpha\left(V(x)-\beta \log \sqrt{1+x^{2}}\right)} d x \leq \int_{-N}^{N} e^{-\alpha\left(V(x)-\beta \log \sqrt{1+x^{2}}\right)} d x+$ $\int_{|x|>N} e^{-\alpha\left(2 / \alpha \log \sqrt{1+x^{2}}\right)} d x \leq 2 N e^{\alpha c}+\int_{|x|>N} \frac{1}{1+x^{2}} d x<\infty$.

Therefore, by Theorem 10 the empirical measures $\hat{\mu}_{n}$ satisfy the large deviation principle with the rate function $\mathbb{I}\left(\nu_{1} \otimes \nu_{2}\right)=\int V(x) \nu_{1}(d x)+\int W(y) \nu_{2}(d y)-$ $\beta \iint \log |x-y| \nu_{1}(d x) \nu_{2}(d y)-I_{0}$. If $W(u)=V(u)=u^{2}$ then $I_{0}=\inf _{x, y}\left\{x^{2}+y^{2}-\right.$ $\beta \log |x-y|\}=\beta / 2(1-\log \beta)$ by calculus.

\section{Auxiliary Results And Proof of Theorem 1}

The proof relies on Varadhan's functional method, see [Ref. 3] Theorem T.1.3], [Ref. [6] Theorem 4.4.10]. It consists of two steps: verification that the Varadhan functional

$$
\Phi \mapsto \mathbb{L}(\Phi):=\lim _{n \rightarrow \infty} \frac{1}{n^{2}} \log E \exp \left(\Phi\left(\hat{\mu}_{n}\right)\right)
$$

is well defined for a large enough class of bounded continuous functions $\Phi: \mathcal{P} \rightarrow \mathbb{R}$, and the proof of exponential tightness of $\left\{\hat{\mu}_{n}\right\}$.

3.1. Varadhan functional. Let $F_{1}, \ldots F_{m}: \mathbb{R}^{2} \rightarrow \mathbb{R}$ be bounded continuous functions. Consider the bounded continuous function $\Phi: \mathcal{P}\left(\mathbb{R}^{2}\right) \rightarrow \mathbb{R}$ given by

$$
\Phi(\mu):=\min _{1 \leq r \leq m} \int F_{r} d \mu .
$$

We will show the following. 
Theorem 2. Under the assumptions of Theorem 1 ,

$$
\begin{array}{r}
\lim _{n \rightarrow \infty} \frac{1}{n^{2}} \log \int \exp \left(n^{2} \Phi\left(\hat{\mu}_{n}\right)\right) f(\mathbf{x}, \mathbf{y}) d \mathbf{x} d \mathbf{y} \\
=\sup \left\{\Phi(\mu)-\int k(x, y) d \mu: \mu=\nu_{1} \otimes \nu_{2} \in \mathcal{P}\left(\mathbb{R}^{2}\right)\right\} .
\end{array}
$$

Denote $\mathbb{K}(\mu)=\int k(x, y) d \mu$. Notice that by Assumption 3 we have $\Phi(\mu)-\mathbb{K}(\mu) \leq$ $\max _{r}\left\|F_{r}\right\|_{\infty}+C$. In particular,

$$
\sup \left\{\Phi(\mu)-\mathbb{K}(\mu): \mu \in \mathcal{P}\left(\mathbb{R}^{2}\right)\right\}<\infty .
$$

We prove (10) as two separate inequalities. It will be convenient to prove the upper bound for a larger class of functions $\Phi$.

Lemma 1. If Assumptions 2 and 3 hold true, then for every bounded continuous function $\Phi: \mathcal{P}\left(\mathbb{R}^{2}\right) \rightarrow \mathbb{R}$ we have

$$
\begin{aligned}
& \limsup _{n \rightarrow \infty} \frac{1}{n^{2}} \log \int \exp \left(n^{2} \Phi\left(\hat{\mu}_{n}\right)\right) f(\mathbf{x}, \mathbf{y}) d \mathbf{x} d \mathbf{y} \\
\leq & \sup \left\{\Phi(\mu)-\mathbb{K}(\mu): \mu=\nu_{1} \otimes \nu_{2} \in \mathcal{P}\left(\mathbb{R}^{2}\right)\right\} .
\end{aligned}
$$

Proof. Notice that for $0<\theta<1$

$$
\begin{gathered}
\int \exp \left(n^{2} \Phi\left(\hat{\mu}_{n}\right)\right) f(\mathbf{x}, \mathbf{y}) d \mathbf{x} d \mathbf{y} \\
=\int \exp \left(n^{2}\left(\Phi\left(\hat{\mu}_{n}\right)-\theta \mathbb{K}\left(\hat{\mu}_{n}\right)\right)-(1-\theta) \sum_{i, j=1}^{n} k\left(x_{i}, y_{j}\right)\right) d \mathbf{x} d \mathbf{y} \\
\leq \exp \left(n^{2} \sup _{\nu_{1}, \nu_{2}}\left(\Phi\left(\nu_{1} \otimes \nu_{2}\right)-\theta \mathbb{K}\left(\nu_{1} \otimes \nu_{2}\right)\right)\right) \int \exp \left(-(1-\theta) \sum_{i, j=1}^{n} k\left(x_{i}, y_{j}\right)\right) d \mathbf{x} d \mathbf{y} .
\end{gathered}
$$

Since

therefore

$$
\sum_{i, j=1}^{n} k\left(x_{i}, y_{j}\right) \geq-n^{2} C+\sum_{j=1}^{n} k\left(x_{j}, y_{j}\right)
$$

$$
\begin{gathered}
\frac{1}{n^{2}} \log \int \exp \left(n^{2} \Phi\left(\hat{\mu}_{n}\right)\right) f(\mathbf{x}, \mathbf{y}) d \mathbf{x} d \mathbf{y} \\
\leq \sup _{\nu_{1}, \nu_{2}}\left\{\Phi\left(\nu_{1} \otimes \nu_{2}\right)-\theta K\left(\nu_{1} \otimes \nu_{2}\right)\right\}+(1-\theta) C+\frac{1}{n} \log M_{1-\theta} .
\end{gathered}
$$

Thus

$$
\begin{gathered}
\limsup _{n \rightarrow \infty} \frac{1}{n^{2}} \log \int \exp \left(n^{2} \Phi\left(\hat{\mu}_{n}\right)\right) f(\mathbf{x}, \mathbf{y}) d \mathbf{x} d \mathbf{y} \\
\leq \sup _{\nu_{1}, \nu_{2}}\left\{\theta\left(\Phi\left(\nu_{1} \otimes \nu_{2}\right)-K\left(\nu_{1} \otimes \nu_{2}\right)\right)+(1-\theta) \Phi\left(\nu_{1} \otimes \nu_{2}\right)\right\}+2(1-\theta) C \\
\leq \theta \sup _{\nu_{1}, \nu_{2}}\left\{\Phi\left(\nu_{1} \otimes \nu_{2}\right)-K\left(\nu_{1} \otimes \nu_{2}\right)\right\}+(1-\theta)\|\Phi\|_{\infty}+2(1-\theta) C .
\end{gathered}
$$

Passing to the limit as $\theta \rightarrow 1$ we get (12).

The proof of the lower bound is a combination of the discretization argument in [Ref. 11 pages 532-535] with the entropy estimate from [Ref. 9] pages 191-192].

Denote by $\mathcal{P}_{0}$ the set of absolutely continuous probability measures $\nu(d x)=$ $f(x) d x$ on $\mathbb{R}$ with compact support supp $(\nu)$, and continuous density $f$. Let us first record the well-known fact. 
Lemma 2. If $\nu \in \mathcal{P}_{0}$ then $\nu$ has finite entropy

$$
H_{f}:=\int \log f(x) \nu(d x)<\infty .
$$

We first establish a weaker version of the lower bound.

Lemma 3. If $\Phi$ is given by (2), then

$$
\begin{array}{r}
\quad \liminf _{n \rightarrow \infty} \frac{1}{n^{2}} \log \int \exp \left(n^{2} \Phi\left(\hat{\mu}_{n}\right)\right) f(\mathbf{x}, \mathbf{y}) d \mathbf{x} d \mathbf{y} \\
\geq \sup \left\{\Phi\left(\nu_{1} \otimes \nu_{2}\right)-\mathbb{K}\left(\nu_{1} \otimes \nu_{2}\right): \nu_{1}, \nu_{2} \in \mathcal{P}_{0}\right\} .
\end{array}
$$

Proof. Fix $\nu_{1}, \nu_{2} \in \mathcal{P}_{0}$. Since $k(x, y) \geq-C$ is bounded from below, $\mathbb{K}\left(\nu_{1} \otimes \nu_{2}\right) \in$ $(-\infty, \infty]$, so without loss of generality we may assume that $k(x, y)$ is $\nu_{1} \otimes \nu_{2}-$ integrable.

Since measures $\nu_{1}, \nu_{2}$ are absolutely continuous and have compact supports, for every integer $n>0$ we can find partitions $\Pi_{1}(n)=\left\{a_{0}<a_{1}<\cdots<a_{n}\right\}$ and $\Pi_{2}(n)=\left\{b_{0}<b_{1}<\cdots<b_{n}\right\}$ of supp $\left(\nu_{1}\right)$, supp $\left(\nu_{2}\right)$ respectively such that

$$
\nu_{1}\left(a_{i-1}, a_{i}\right)=\nu_{2}\left(b_{j-1}, b_{j}\right)=\frac{1}{n} \text { for } i, j=1,2, \ldots, n .
$$

Then, denoting $A=\left[a_{0}, a_{1}\right] \times\left[a_{1}, a_{2}\right] \times \cdots \times\left[a_{n-1}, a_{n}\right]$ and $B=\left[b_{0}, b_{1}\right] \times\left[b_{1}, b_{2}\right] \times$ $\cdots \times\left[b_{n-1}, b_{n}\right]$, we have

$$
\begin{gathered}
\int \exp \left(n^{2} \Phi\left(\hat{\mu}_{n}\right)\right) f(\mathbf{x}, \mathbf{y}) d \mathbf{x} d \mathbf{y} \\
\geq \int_{A \times B} \exp \left(\min _{r} \sum_{i, j=1}^{n} F_{r}\left(x_{i}, y_{j}\right)-\sum_{i, j=1}^{n} k\left(x_{i}, y_{j}\right)\right) d \mathbf{x} d \mathbf{y} .
\end{gathered}
$$

Write $\nu_{1}=f(x) d x, \nu_{2}=g(y) d y$. By our choice of the partitions, functions

$$
f_{i}(x):=n f(x) I_{\left[a_{i-1}, a_{i}\right]}
$$

and

$$
g_{j}(x):=n g(x) I_{\left[b_{j-1}, b_{j}\right]}
$$

are probability densities. Let

$$
S(\mathbf{x}, \mathbf{y})=\min _{r} \sum_{i, j=1}^{n} F_{r}\left(x_{i}, y_{j}\right)-\sum_{i, j=1}^{n} k\left(x_{i}, y_{j}\right)-\sum_{i=1}^{n} \log f\left(x_{i}\right)-\sum_{j=1}^{n} \log g\left(y_{j}\right) .
$$

Integrating over a smaller set $\left\{f_{1}\left(x_{1}\right)>0, \ldots, f_{n}\left(x_{n}\right)>0, g_{1}\left(y_{1}\right)>0, \ldots, g_{n}\left(y_{n}\right)>\right.$ $0\}$ on the right hand side of (14) we get

$$
\begin{gathered}
\int \exp \left(n^{2} \Phi\left(\hat{\mu}_{n}\right)\right) f(\mathbf{x}, \mathbf{y}) d \mathbf{x} d \mathbf{y} \\
\geq \frac{1}{n^{2 n}} \int \exp (S(\mathbf{x}, \mathbf{y})) \prod_{i=1}^{n} f_{i}\left(x_{i}\right) \prod_{j=1}^{n} g_{j}\left(y_{j}\right) d \mathbf{x} d \mathbf{y} .
\end{gathered}
$$

Using Jensen's inequality, applied to the convex exponential function in the last integral, we get

$$
\int \exp \left(n^{2} \Phi\left(\hat{\mu}_{n}\right)\right) f(\mathbf{x}, \mathbf{y}) d \mathbf{x} d \mathbf{y} \geq \frac{1}{n^{2 n}} \exp \left(S_{1}-S_{2}-S_{3}-S_{4}\right),
$$


where

$$
\begin{aligned}
S_{1} & =\int_{\mathbb{R}^{2 n}}\left(\min _{r} \sum_{i, j=1}^{n} F_{r}\left(x_{i}, y_{j}\right)\right) \prod_{i=1}^{n} f_{i}\left(x_{i}\right) \prod_{j=1}^{n} g_{j}\left(x_{j}\right) d \mathbf{x} d \mathbf{y}, \\
S_{2} & =\int_{\mathbb{R}^{2 n}} \sum_{i, j=1}^{n} k\left(x_{i}, y_{j}\right) \prod_{i=1}^{n} f_{i}\left(x_{i}\right) \prod_{j=1}^{n} g_{j}\left(y_{j}\right) d \mathbf{x} d \mathbf{y}, \\
S_{3} & =\int_{\mathbb{R}^{n}} \sum_{i=1}^{n} \log f\left(x_{i}\right) \prod_{i=1}^{n} f_{i}\left(x_{i}\right) d \mathbf{x}, \\
S_{4} & =\int_{\mathbb{R}^{n}} \sum_{j=1}^{n} \log g\left(y_{j}\right) \prod_{j=1}^{n} g_{j}\left(y_{j}\right) d \mathbf{y} .
\end{aligned}
$$

We need the following identities. (Proofs of all Claims are postponed until the end of this proof.)

Claim 1. For a $\nu_{1} \otimes \nu_{2}$-integrable function $h$, we have

$$
\begin{array}{r}
\int_{\mathbb{R}^{n}} \sum_{j=1}^{n} h\left(y_{j}\right) \prod_{j=1}^{n} g_{j}\left(y_{j}\right) d \mathbf{y}=n \int_{\mathbb{R}} h(y) g(y) d y, \\
\int_{\mathbb{R}^{n}} \sum_{i=1}^{n} h\left(x_{i}\right) \prod_{i=1}^{n} f_{i}\left(x_{i}\right) d \mathbf{x}=n \int_{\mathbb{R}} h(x) f(x) d x, \\
\int_{\mathbb{R}^{2 n}} \sum_{i, j=1}^{n} h\left(x_{i}, y_{j}\right) \prod_{i=1}^{n} f_{i}\left(x_{i}\right) \prod_{j=1}^{n} g_{j}\left(y_{j}\right) d \mathbf{x} d \mathbf{y} \\
=n^{2} \iint h(x, y) f(x) g(y) d x d y .
\end{array}
$$

Lemma 2 says that the entropies $H_{f}=\int \log f(x) f(x) d x, H_{g}=\int \log g(y) g(y) d y$ are finite. Thus the functions $k(x, y), \log f(x)$, and $\log g(y)$ are $\nu_{1} \otimes \nu_{2}$-integrable. Applying Claim 1 we get $S_{2}=n^{2} \mathbb{K}\left(\nu_{1} \otimes \nu_{2}\right), S_{3}=n H_{f}$, and $S_{4}=n H_{g}$. Therefore,

$$
\begin{gathered}
\int \exp \left(n^{2} \Phi\left(\hat{\mu}_{n}\right)\right) f(\mathbf{x}, \mathbf{y}) d \mathbf{x} d \mathbf{y} \\
\geq \frac{1}{n^{2 n}} \exp \left(S_{1}-n^{2} \mathbb{K}\left(\nu_{1} \otimes \nu_{2}\right)-n H_{f}-n H_{g}\right) .
\end{gathered}
$$

We need the following lower bound for $S_{1}$.

\section{Claim 2.}

$$
\begin{array}{r}
\int\left(\min _{r} \sum_{i, j=1}^{n} F_{r}\left(x_{i}, y_{j}\right)\right) \prod f_{i}\left(x_{i}\right) \prod g_{j}\left(y_{j}\right) d \mathbf{x} d \mathbf{y} \\
\geq \min _{r} \sum_{i, j=1}^{n} F_{r,(i, j)}
\end{array}
$$

where

$$
F_{r,(i, j)}=\min \left\{F_{r}(x, y): a_{i-1} \leq x \leq a_{i}, b_{j-1} \leq y \leq b_{j}\right\}
$$


Combining inequalities (15) and (16), we get

$$
\begin{array}{r}
\frac{1}{n^{2}} \log \int \exp \left(n^{2} \Phi\left(\hat{\mu}_{n}\right)\right) f(\mathbf{x}, \mathbf{y}) d \mathbf{x} d \mathbf{y} \\
\geq \frac{1}{n^{2}} \min _{r} \sum_{i, j=1}^{n} F_{r,(i, j)}-\mathbb{K}\left(\nu_{1} \otimes \nu_{2}\right)-\frac{1}{n} H_{f}-\frac{1}{n} H_{g}-\frac{2}{n} \log n .
\end{array}
$$

Since functions $F_{r}(x, y)$ are continuous and $\nu_{1}, \nu_{2}$ have compact support and continuous densities $f, g$, therefore $\nu_{1} \otimes \nu_{2}$-almost surely $\sum_{i, j=1}^{n} F_{r,(i, j)} I_{\left(a_{i-1}, a_{i}\right)}(x) I_{\left(b_{j-1}, b_{j}\right)}(y) \rightarrow$ $F_{r}(x, y)$ (to see this, notice that for fixed $\varepsilon>0$, the sequence convergences for all $(x, y)$ such that $f(x) \geq \varepsilon, g(y) \geq \varepsilon$.), and the functions are bounded. Since $1 \leq r \leq m$ ranges over a finite set of values only we have

$$
\begin{gathered}
\lim _{n \rightarrow \infty} \frac{1}{n^{2}} \min _{r} \sum_{i, j=1}^{n} F_{r,(i, j)} \\
=\min _{r} \lim _{n \rightarrow \infty} \sum_{i, j=1}^{n} F_{r,(i, j)} \nu_{1}\left(a_{i-1}, a_{i}\right) \nu_{2}\left(b_{j-1} b_{j}\right)=\Phi\left(\nu_{1} \otimes \nu_{2}\right) .
\end{gathered}
$$

Letting $n \rightarrow \infty$ in (17) we obtain (13).

To conclude the proof, it remains to prove Claims 1 and 2

Proof of Claim 1 Switching the order of integration and summation, we get

$$
\begin{aligned}
& \int \sum_{j=1}^{n} h\left(y_{j}\right) \prod g_{j}\left(y_{j}\right) d \mathbf{y}=\sum_{j=1}^{n} \int h\left(y_{j}\right) g_{j}\left(y_{j}\right) d y_{j} \prod_{i \neq j} \int g_{i}\left(y_{i}\right) d y_{i} \\
= & \sum_{j=1}^{n} \int h\left(y_{j}\right) g_{j}\left(y_{j}\right) d y_{j}=n \sum_{j=1}^{n} \int_{b_{j-1}}^{b_{j}} h(y) g(y) d y=n \int_{b_{0}}^{b_{n}} h(y) g(y) d y .
\end{aligned}
$$

The other two identities follow by a similar argument.

Proof of Claim 2, Fix $0 \leq k \leq n, x_{1}, \ldots, x_{k} \in \mathbb{R}$ and $y_{1}, \ldots, y_{n} \in \mathbb{R}$. Let

$$
G_{r, k}\left(x_{1}, \ldots, x_{k}\right):=\sum_{i=1}^{k} \sum_{j=1}^{n} F_{r}\left(x_{i}, y_{j}\right)+\sum_{i=k+1}^{n} \sum_{j=1}^{n} \min _{a_{i-1} \leq x \leq a_{i}} F_{r}\left(x, y_{j}\right) .
$$

If $a_{k-1}<x_{k}<a_{k}$, we have

$$
\begin{gathered}
\min _{r} G_{r, k}\left(x_{1}, \ldots, x_{k}\right)= \\
\min _{r}\left(\sum_{i=1}^{k-1} \sum_{j} F_{r}\left(x_{i}, y_{j}\right)+\sum_{j} F_{r}\left(x_{k}, y_{j}\right)+\sum_{i=k+1}^{n} \sum_{j} \min _{a_{i-1} \leq x \leq a_{i}} F_{r}\left(x, y_{j}\right)\right) \\
\geq \min _{r} G_{r, k-1}\left(x_{1}, \ldots, x_{k-1}\right) .
\end{gathered}
$$

Therefore,

$$
\int_{a_{k-1}}^{a_{k}} \min _{r} G_{r, k}(\mathbf{x}) f_{k}\left(x_{k}\right) d x_{k} \geq \min _{r} G_{r, k-1}(\mathbf{x})
$$

Recurrently,

$$
\int \min _{r}\left(\sum_{i=1}^{n} \sum_{j=1}^{n} F_{r}\left(x_{i}, y_{j}\right)\right) \prod f_{i}\left(x_{i}\right) d \mathbf{x}
$$




$$
\begin{gathered}
=\int \min _{r} G_{r, n}(\mathbf{x}) \prod f_{i}\left(x_{i}\right) d \mathbf{x} \geq \min _{r} G_{r, 0}(\mathbf{x}) \\
=\min _{r}\left(\sum_{i=1}^{n} \sum_{j=1}^{n} \min _{a_{i-1} \leq x \leq a_{i}} F_{r}\left(x, y_{j}\right)\right) .
\end{gathered}
$$

Applying the same reasoning to variables $y_{1}, \ldots, y_{n}$ and

$$
G_{r, k}\left(y_{1}, \ldots, y_{k}\right):=\sum_{j=1}^{k} \sum_{i=1}^{n} \min _{a_{i-1} \leq x \leq a_{i}} F_{r}\left(x, y_{j}\right)+\sum_{j=k+1}^{n} \sum_{i=1}^{n} F_{r,(i, j)}
$$

we get (16).

This concludes the proof.

The next Lemmas show that the right hand sides of (12) and (13) coincide.

Let $\mathcal{P}_{c}$ denote compactly supported probability measures.

Lemma 4. If Assumption 3 holds true, then

$$
\begin{aligned}
& \sup \left\{\Phi\left(\nu_{1} \otimes \nu_{2}\right)-\mathbb{K}\left(\nu_{1} \otimes \nu_{2}\right): \nu_{1}, \nu_{2} \in \mathcal{P}_{c}\right\} \\
= & \sup \left\{\Phi\left(\nu_{1} \otimes \nu_{2}\right)-\mathbb{K}\left(\nu_{1} \otimes \nu_{2}\right): \nu_{1}, \nu_{2} \in \mathcal{P}\right\} .
\end{aligned}
$$

Proof. Clearly the left-hand side of (18) cannot exceed the right hand side. To show the converse inequality, fix $\eta>0$ and $\nu_{1}, \nu_{2} \in \mathcal{P}$ such that

$$
\Phi\left(\nu_{1} \otimes \nu_{2}\right)-\mathbb{K}\left(\nu_{1} \otimes \nu_{2}\right) \geq \sup _{\nu_{1}, \nu_{2} \in \mathcal{P}}\left\{\Phi\left(\nu_{1} \otimes \nu_{2}\right)-\mathbb{K}\left(\nu_{1} \otimes \nu_{2}\right)\right\}-\eta .
$$

Since the supremum is finite, see (11), and $k(x, y)$ is bounded from below, therefore $\iint|k(x, y)| d \nu_{1} d \nu_{2}<\infty$.

For $L>0$ large enough, define probability measures $\nu_{j, L}$ by

$$
\nu_{j, L}(A):=\frac{\nu_{j}(A \cap[-L, L])}{\nu_{j}([-L, L])}, j=1,2 .
$$

By definition, measures $\nu_{j, L} \in \mathcal{P}_{c}$ have compact support. Since $-C \leq$ $k(x, y) I_{|x|<L,|y|<L} \leq|k(x, y)|$ and $k$ is $\nu_{1} \otimes \nu_{2}$-integrable, by Lebesgue's dominated convergence theorem

$$
\lim _{L \rightarrow \infty} \mathbb{K}\left(\nu_{1, L} \otimes \nu_{2, L}\right)=\frac{\lim _{L \rightarrow \infty} \int_{-L}^{L} \int_{-L}^{L} k(x, y) \nu_{1}(d x) \nu_{2}(d y)}{\lim _{L \rightarrow \infty} \nu_{1}([-L, L]) \nu_{2}([-L, L])}=\mathbb{K}\left(\nu_{1} \otimes \nu_{2}\right) .
$$

Similarly,

$$
\lim _{L \rightarrow \infty} \Phi\left(\nu_{1, L} \otimes \nu_{2, L}\right)=\Phi\left(\nu_{1} \otimes \nu_{2}\right)
$$

Thus (18) follows.

Lemma 5. If Assumptions 3 and 5 hold true, then

$$
\begin{aligned}
& \sup \left\{\Phi\left(\nu_{1} \otimes \nu_{2}\right)-\mathbb{K}\left(\nu_{1} \otimes \nu_{2}\right): \nu_{1}, \nu_{2} \in \mathcal{P}_{0}\right\} \\
= & \sup \left\{\Phi\left(\nu_{1} \otimes \nu_{2}\right)-\mathbb{K}\left(\nu_{1} \otimes \nu_{2}\right): \nu_{1}, \nu_{2} \in \mathcal{P}\right\} .
\end{aligned}
$$

Proof. Trivially,

$$
\sup _{\nu_{1}, \nu_{2} \in \mathcal{P}_{0}}\left\{\Phi\left(\nu_{1} \otimes \nu_{2}\right)-\mathbb{K}\left(\nu_{1} \otimes \nu_{2}\right)\right\} \leq \sup _{\nu_{1}, \nu_{2} \in \mathcal{P}}\left\{\Phi\left(\nu_{1} \otimes \nu_{2}\right)-\mathbb{K}\left(\nu_{1} \otimes \nu_{2}\right)\right\} .
$$


To show the converse inequality, fix $\eta>0$ and compactly supported $\nu_{1}, \nu_{2} \in \mathcal{P}_{c}$ such that

$$
\Phi\left(\nu_{1} \otimes \nu_{2}\right)-\mathbb{K}\left(\nu_{1} \otimes \nu_{2}\right) \geq \sup _{\nu_{1}, \nu_{2} \in \mathcal{P}}\left\{\Phi\left(\nu_{1} \otimes \nu_{2}\right)-\mathbb{K}\left(\nu_{1} \otimes \nu_{2}\right)\right\}-\eta,
$$

see Lemma 4 As previously, $k(x, y)$ is $\nu_{1} \otimes \nu_{2}$-integrable, see (11).

Consider the convolution $\nu_{j}^{\varepsilon}(A):=\frac{1}{2 \varepsilon} \int_{-\varepsilon}^{\varepsilon} \nu_{j}(A-x) d x$, where $j=1,2$ and $0<$ $\varepsilon \leq 1$. Measures $\nu_{1}^{\varepsilon}, \nu_{2}^{\varepsilon}$ have continuous densities, and since $\nu_{1}, \nu_{2}$ have compact supports, $\nu_{1}^{\varepsilon}, \nu_{2}^{\varepsilon}$ also have compact support. Thus $\nu_{1}^{\varepsilon}, \nu_{2}^{\varepsilon} \in \mathcal{P}_{0}$ and

$$
\sup _{\nu_{1}, \nu_{2} \in \mathcal{P}_{0}}\left\{\Phi\left(\nu_{1} \otimes \nu_{2}\right)-\mathbb{K}\left(\nu_{1} \otimes \nu_{2}\right)\right\} \geq \Phi\left(\nu_{1}^{\varepsilon} \otimes \nu_{2}^{\varepsilon}\right)-\mathbb{K}\left(\nu_{1}^{\varepsilon} \otimes \nu_{2}^{\varepsilon}\right) .
$$

As $\varepsilon \rightarrow 0$ measure $\nu_{j}^{\varepsilon}$ converges weakly to $\nu_{j}$. Hence

$$
\lim _{\varepsilon \rightarrow 0} \Phi\left(\nu_{1}^{\varepsilon} \otimes \nu_{2}^{\varepsilon}\right)=\Phi\left(\nu_{1} \otimes \nu_{2}\right) .
$$

Assumption [5asserts that $V(x, y):=\beta \log |x-y|+k(x, y)$ is a continuous function. Thus $|V(x, y)|$ is bounded on the compact set $\operatorname{supp}\left(\nu_{1}^{1} \otimes \nu_{2}^{1}\right)$. Since the supports of $\nu_{1}^{\varepsilon} \otimes \nu_{2}^{\varepsilon}$ are contained in $\operatorname{supp}\left(\nu_{1}^{1} \otimes \nu_{2}^{1}\right)$, and $\nu_{j}^{\varepsilon} \rightarrow \nu_{j}$, we get

$$
\iint V(x, y) \nu_{1}^{\varepsilon}(d x) \nu_{2}^{\varepsilon}(d y) \rightarrow \iint V(x, y) \nu_{1}(d x) \nu_{2}(d y) .
$$

This concludes the proof if $\beta=0$. If $\beta>0$, then $\log |x-y|$ is $\nu_{1} \otimes \nu_{2}$-integrable as a linear combination of integrable functions, $\log |x-y|=(V(x, y)-k(x, y)) / \beta$. Therefore we have

$$
\begin{gathered}
\Phi\left(\nu_{1}^{\varepsilon} \otimes \nu_{2}^{\varepsilon}\right)-\mathbb{K}\left(\nu_{1}^{\varepsilon} \otimes \nu_{2}^{\varepsilon}\right)=\Phi\left(\nu_{1}^{\varepsilon} \otimes \nu_{2}^{\varepsilon}\right) \\
-\iint V(x, y) \nu_{1}^{\varepsilon}(d x) \nu_{2}^{\varepsilon}(d y)+\beta \iint \log |x-y| \nu_{1}(d x) \nu_{2}(d y) \\
-\beta\left(\iint \log |x-y| \nu_{1}(d x) \nu_{2}(d y)-\iint \log |x-y| \nu_{1}^{\varepsilon}(d x) \nu_{2}^{\varepsilon}(d y)\right) .
\end{gathered}
$$

Taking the lim sup as $\varepsilon \rightarrow 0$, from (22), (23), (24), and (21) we get

$$
\begin{aligned}
& \sup _{\nu_{1}, \nu_{2} \in \mathcal{P}_{0}}\left\{\Phi\left(\nu_{1} \otimes \nu_{2}\right)-\mathbb{K}\left(\nu_{1} \otimes \nu_{2}\right)\right\} \geq \sup _{\nu_{1}, \nu_{2} \in \mathcal{P}}\left\{\Phi\left(\nu_{1} \otimes \nu_{2}\right)-\mathbb{K}\left(\nu_{1} \otimes \nu_{2}\right)\right\}-\eta \\
& -\limsup _{\varepsilon \rightarrow 0}\left(\iint \log |x-y| \nu_{1}(d x) \nu_{2}(d y)-\iint \log |x-y| \nu_{1}^{\varepsilon}(d x) \nu_{2}^{\varepsilon}(d y)\right) .
\end{aligned}
$$

Since $\eta>0$ is arbitrary, to end the proof we use the following.

\section{Claim 3.}

$$
\limsup _{\varepsilon \rightarrow 0}\left(\iint \log |x-y| \nu_{1}(d x) \nu_{2}(d y)-\iint \log |x-y| \nu_{1}^{\varepsilon}(d x) \nu_{2}^{\varepsilon}(d y)\right) \leq 0 .
$$

Proof of Claim 3. Claim 3] is established by the argument in [Ref. 9, pages 192-193]. For completeness, we repeat it here. Let $X, Y$ be independent random variables with distributions $\nu_{1}, \nu_{2}$ respectively and let $Z=X-Y$. Since $\log |Z|$ is integrable, $\operatorname{Pr}(Z=0)=0$. Let $U \in[-2,2]$ be a r. v. independent of $Z$ with 
the density $f(u)=(2-|u|) / 4$. It is easy to see that $\iint \log |x-y| \nu_{1}^{\varepsilon}(d x) \nu_{2}^{\varepsilon}(d y)=$ $E \log |Z+\varepsilon U|$, and the inequality to prove reads

$$
\limsup _{\varepsilon \rightarrow 0} E\left(\log ^{+} \frac{1}{\left|1+\varepsilon \frac{U}{Z}\right|}\right) \leq 0 .
$$

For fixed $z \neq 0$ we have

$$
E\left(\log ^{+} \frac{1}{\left|1+\varepsilon \frac{U}{z}\right|}\right) \leq \frac{1}{\log 2} \log \left(1+\frac{2 \varepsilon}{|z|}\right) .
$$

Indeed, since $(2-|u|) / 4 \leq 1 / 2$ we get

$$
E\left(\log ^{+} \frac{1}{\left|1+\varepsilon \frac{U}{z}\right|}\right) \leq \frac{|z|}{4 \varepsilon} \int_{1-2 \varepsilon /|z|}^{1+2 \varepsilon /|z|} \log ^{+} \frac{1}{|x|} d x .
$$

Therefore,

$$
E\left(\log ^{+} \frac{1}{\left|1+\varepsilon \frac{U}{z}\right|}\right) \leq\left\{\begin{array}{ll}
\frac{|z|}{4 \varepsilon} \int_{1-2 \varepsilon /|z|}^{1} \log \frac{1}{x} d x & \text { if }|z|>2 \varepsilon \\
\frac{|z|}{4 \varepsilon}\left(\int_{0}^{1} \log \frac{1}{x} d x+\int_{0}^{2 \varepsilon /|z|-1} \log ^{+} \frac{1}{x} d x\right) & \text { if }|z| \leq 2 \varepsilon
\end{array} .\right.
$$

If $|z|>2 \varepsilon$ we get $E\left(\log ^{+} \frac{1}{\left|1+\varepsilon \frac{U}{z}\right|}\right) \leq \frac{1}{2} \log \frac{1}{1-2 \varepsilon /|z|}<\frac{1}{2} \log \left(1+\frac{2 \varepsilon}{|z|}\right) \leq \frac{1}{\log 2} \log (1+$ $\left.\frac{2 \varepsilon}{|z|}\right)$. If $|z| \leq 2 \varepsilon$, then $E\left(\log ^{+} \frac{1}{\left|1+\varepsilon \frac{U}{z}\right|}\right) \leq \frac{|z|}{2 \varepsilon} \int_{0}^{1} \log \frac{1}{x} d x \leq 1 \leq \frac{1}{\log 2} \log \left(1+\frac{2 \varepsilon}{|z|}\right)$.

Thus in both cases, 250lds true.

To finish the proof we integrate inequality (25) and get

$$
\limsup _{\varepsilon \rightarrow 0} E\left(\log ^{+} \frac{1}{\left|1+\varepsilon \frac{U}{Z}\right|}\right) \leq \frac{1}{\log 2} \limsup _{\varepsilon \rightarrow 0} E(\log (1+2 \varepsilon /|Z|)) .
$$

For $\varepsilon<1 / 2$ we have $\log (1+2 \varepsilon /|Z|) \leq \log 2+\log ^{+} \frac{1}{|Z|}$ and $\log ^{+} \frac{1}{|Z|}$ is integrable. Lebesgue's dominated convergence theorem yields

$$
\limsup _{\varepsilon \rightarrow 0} E(\log (1+2 \varepsilon /|Z|))=0 .
$$

Proof of Theorem 2, Combining Lemmas 1 and 3 we have

$$
\begin{aligned}
& \sup \left\{\Phi\left(\nu_{1} \otimes \nu_{2}\right)-\mathbb{K}\left(\nu_{1} \otimes \nu_{2}\right): \nu_{1}, \nu_{2} \in \mathcal{P}_{0}\right\} \\
\leq & \liminf _{n \rightarrow \infty} \frac{1}{n^{2}} \log \int \exp \left(n^{2} \Phi\left(\hat{\mu}_{n}\right)\right) f(\mathbf{x}, \mathbf{y}) d \mathbf{x} d \mathbf{y} \\
\leq & \limsup _{n \rightarrow \infty} \frac{1}{n^{2}} \log \int \exp \left(n^{2} \Phi\left(\hat{\mu}_{n}\right)\right) f(\mathbf{x}, \mathbf{y}) d \mathbf{x} d \mathbf{y} \\
\leq & \sup \left\{\Phi\left(\nu_{1} \otimes \nu_{2}\right)-\mathbb{K}\left(\nu_{1} \otimes \nu_{2}\right): \nu_{1}, \nu_{2} \in \mathcal{P}\right\} .
\end{aligned}
$$

By (20), all of the above inequalities are in fact equalities. Thus (10) holds true. 
3.2. Exponential tightness. Recall that $\left\{\hat{\mu}_{n}\right\}$ is exponentially tight if for every $m>0$ there is a compact subset $\mathcal{K} \subset \mathcal{P}$ such that

$$
\sup _{n} \frac{1}{n^{2}} \log \operatorname{Pr}\left(\hat{\mu}_{n} \notin \mathcal{K}\right)<-m .
$$

Our proof of exponential tightness is a concrete implementation of de Acosta. (5)

Assumption 3 implies that $k(x, y)+C \geq 0$. Let $q: \mathcal{P}\left(\mathbb{R}^{2}\right) \rightarrow[0, \infty]$ be given by

$$
q(\mu)=\int_{\mathbb{R}^{2}}(k(x, y)+C) d \mu .
$$

Lemma 6. If Assumptions 3 , 4 hold true, then $q$ has pre-compact level sets: for every $t>0, q^{-1}[0, t]$ is a pre-compact set in $\mathcal{P}$.

Proof. Fix $t>0$ and denote $\mathcal{K}:=\left\{\mu \in \mathcal{P}\left(\mathbb{R}^{2}\right): q(\mu) \leq t\right\}$. We will show that $\mathcal{K}$ is pre-compact.

Assumption 4 says that for every $\varepsilon>0$ the set $K_{\varepsilon}:=\{(x, y): C+k(x, y) \leq t / \varepsilon\}$ is a compact subset of $\mathbb{R}^{2}$. For every $\mu \in \mathcal{K}$ by Chebyshev's inequality we have

$$
\mu\left(K_{\varepsilon}^{c}\right) \leq \mu(\{(x, y): C+k(x, y)>t / \varepsilon\}) \leq \frac{\varepsilon q(\mu)}{t}=\varepsilon .
$$

Thus $\mathcal{K}$ is pre-compact, and its weak closure $\overline{\mathcal{K}}$ is compact.

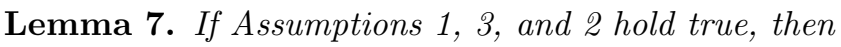

$$
\sup _{n} \frac{1}{n^{2}} \log \int \exp \left(\frac{1}{2} n^{2} q\left(\hat{\mu}_{n}\right)\right) f(\mathbf{x}, \mathbf{y}) d \mathbf{x} d \mathbf{y}<\infty .
$$

Proof. We have

$$
\begin{gathered}
\int \exp \left(\frac{1}{2} n^{2} q\left(\hat{\mu}_{n}\right)\right) f(\mathbf{x}, \mathbf{y}) d \mathbf{x} d \mathbf{y}=\int \exp \left(\frac{1}{2} n^{2} C-\frac{1}{2} \sum_{i, j=1}^{n} k\left(x_{i}, y_{j}\right)\right) d \mathbf{x} d \mathbf{y} \\
\leq e^{\frac{1}{2} n^{2} C} \int \prod_{i, j=1}^{n} \sqrt{g\left(x_{i}, y_{j}\right)} d \mathbf{x} d \mathbf{y} \leq e^{n^{2} C} M_{1 / 2}^{n} .
\end{gathered}
$$

Therefore the left-hand side of (26) is at most $C+\log ^{+} M_{1 / 2}<\infty$.

Theorem 3. Under the assumptions of Theorem 1, the sequence $\left\{\hat{\mu}_{n}\right\}$ is exponentially tight.

Proof. Notice that by (10) used with $\Phi(\mu):=0$ we have $\frac{1}{n^{2}} \log Z_{n} \rightarrow L_{0}:=$ $-\inf _{\mu} \int k(x, y) d \mu=-\inf _{x, y} k(x, y)$. Since $L_{0}$ is finite, see (11), therefore by Lemma 7 we have

$$
\sup _{n} \frac{1}{n^{2}} \log \int \exp \left(\frac{1}{2} n^{2} q\left(\hat{\mu}_{n}\right)\right) \frac{1}{Z_{n}} f(\mathbf{x}, \mathbf{y}) d \mathbf{x} d \mathbf{y}=C_{1}<\infty .
$$

Fix $m>0$. Let $\mathcal{K} \subset \mathcal{P}$ be the pre-compact set from Lemma 6 corresponding to $t=2 m+2 C_{1}$.

Applying Chebyshev's inequality to probability measure (3) we get

$$
\operatorname{Pr}\left(\hat{\mu}_{n} \notin \overline{\mathcal{K}}\right) \leq \operatorname{Pr}\left(\hat{\mu}_{n} \notin \mathcal{K}\right)=\operatorname{Pr}\left(q\left(\hat{\mu}_{n}\right)>t\right) \leq e^{-\frac{1}{2} n^{2} t} \int \exp \left(\frac{1}{2} n^{2} q\left(\hat{\mu}_{n}\right)\right) d \operatorname{Pr} .
$$

Therefore

$$
\operatorname{Pr}\left(\hat{\mu}_{n} \notin \overline{\mathcal{K}}\right) \leq e^{-\frac{1}{2} n^{2} t} e^{n^{2} C_{1}},
$$


and

for all $n$.

$$
\frac{1}{n^{2}} \log \operatorname{Pr}\left(\hat{\mu}_{n} \notin \overline{\mathcal{K}}\right) \leq-t / 2+C_{1}=-m
$$

Proof of Theorem 1, Recall that the space $\mathcal{P}=\mathcal{P}\left(\mathbb{R}^{2}\right)$ of probability measures on $\mathbb{R}^{2}$ with the topology of weak convergence is a Polish space. By Theorem 3 . $\left\{\hat{\mu}_{n}\right\}$ is exponentially tight. Theorem 2 says that the Varadhan functional

$$
\mathbb{L}(\Phi):=\lim _{n \rightarrow \infty} \frac{1}{n^{2}} \log E\left(\exp n^{2} \Phi\left(\hat{\mu}_{n}\right)\right)
$$

is defined on all functions $\Phi$ given by (9), and

$$
\begin{aligned}
\mathbb{L}(\Phi) & =\sup _{\nu_{1}, \nu_{2}}\left\{\Phi\left(\nu_{1} \otimes \nu_{2}\right)-\mathbb{K}\left(\nu_{1} \otimes \nu_{2}\right)\right\}-\lim _{n \rightarrow \infty} \frac{1}{n^{2}} \log Z_{n} \\
& =\sup _{\nu_{1}, \nu_{2}}\left\{\Phi\left(\nu_{1} \otimes \nu_{2}\right)-\mathbb{K}\left(\nu_{1} \otimes \nu_{2}\right)\right\}+\inf _{x, y} k(x, y) .
\end{aligned}
$$

Thus

$$
\mathbb{L}(\Phi)=\sup _{\nu_{1}, \nu_{2}}\left\{\Phi\left(\nu_{1} \otimes \nu_{2}\right)-\mathbb{K}\left(\nu_{1} \otimes \nu_{2}\right)\right\}+I_{0} .
$$

Functions $\Phi$ defined by (9) form a subset of $C_{b}\left(\mathcal{P}\left(\mathbb{R}^{2}\right)\right)$ which separates points of $\mathcal{P}\left(\mathbb{R}^{2}\right)$ and is closed under the operation of taking pointwise minima. Thus by [Ref. 3. Theorem T.1.3] or [Ref. [6. Theorem 4.4.10], the empirical measures $\left\{\hat{\mu}_{n}\right\}$ satisfy the large deviation principle with the rate function

$$
\mathbb{I}(\mu):=\sup \{\Phi(\mu)-\mathbb{L}(\Phi)\}
$$

here, the supremum is taken over all $F_{1}, \ldots, F_{m} \in C_{b}\left(\mathbb{R}^{2}\right)$ and $\Phi(\mu)$ is defined by (9).

It remains to prove formula (44). Fix $\nu_{1}, \nu_{2} \in \mathcal{P}$. From (27), for $\Phi$ given by (9) we have $\mathbb{L}(\Phi) \geq \Phi\left(\nu_{1} \otimes \nu_{2}\right)-\mathbb{K}\left(\nu_{1} \otimes \nu_{2}\right)+I_{0}$. Thus formula (28) implies that

$$
\mathbb{I}\left(\nu_{1} \otimes \nu_{2}\right) \leq \mathbb{K}\left(\nu_{1} \otimes \nu_{2}\right)-I_{0} .
$$

To prove the converse inequality we use the fact that we already know that the large deviations principle holds. The large deviations principle implies that

$$
\mathbb{I}\left(\nu_{1} \otimes \nu_{2}\right)=\sup _{\Phi \in C_{b}(\mathcal{P})}\left\{\Phi\left(\nu_{1} \otimes \nu_{2}\right)-\mathbb{L}(\Phi)\right\} .
$$

Now consider $\Phi_{M}(\mu)=\int(M \wedge k(x, y)) d \mu$. Assumptions 3 and $[5$ imply that $(x, y) \mapsto M \wedge k(x, y)$ is a bounded continuous function for every real $M$. Thus $\Phi_{M}$ is given by (9). Since $M \wedge k(x, y) \leq k(x, y)$, from (27) we get $\mathbb{L}\left(\Phi_{M}\right) \leq I_{0}$. Thus

$$
\mathbb{I}\left(\nu_{1} \otimes \nu_{2}\right) \geq \sup _{M}\left\{\Phi_{M}\left(\nu_{1} \otimes \nu_{2}\right)-\mathbb{L}\left(\Phi_{M}\right)\right\} \geq \limsup _{M \rightarrow \infty} \int M \wedge k(x, y) d \mu-I_{0} .
$$

This together with (29) proves (4) for product measures.

It remains to verify that if $\mu_{0}$ is not a product measure, then $\mathbb{I}\left(\mu_{0}\right)=\infty$. To this end, take bounded continuous functions $F(x), G(y)$ such that

$$
\delta:=\int F(x) G(y) \mu_{0}(d x, d y)-\int F(x) \mu_{0}(d x, d y) \int G(y) \mu_{0}(d x, d y)>0 .
$$

For $b>0$, let

$$
\Phi_{b}(\mu):=b\left(\int F(x) G(y) \mu(d x, d y)-\int F(x) \mu(d x, d y) \int G(y) \mu(d x, d y)\right) .
$$


Clearly, $\Phi_{b}: \mathcal{P} \rightarrow \mathbb{R}$ is a bounded continuous function, which vanishes on product measures. By the upper bound (12) we therefore have $\mathbb{L}\left(\Phi_{b}\right) \leq I_{0}$. So $\mathbb{I}\left(\mu_{0}\right) \geq$ $\Phi_{b}\left(\mu_{0}\right)-\mathbb{L}\left(\Phi_{b}\right) \geq b \delta-I_{0}$. Since $b$ can be arbitrarily large, $\mathbb{I}\left(\mu_{0}\right)=\infty$.

Acknowledgements. I would like to thank P. Dupuis for a conversation on nonconvex rate functions.

\section{REFERENCES}

[1] Ben Arous, G. \& Guionnet, A. (1997). Large deviations for Wigner's law and Voiculescu's non-commutative entropy. Probab. Theory Related Fields, 108(4), 517-542.

[2] Berg, C., Christensen, J. P. R., \& Ressel, P. (1984). Harmonic analysis on semigroups. New York: Springer-Verlag.

[3] Bryc, W. (1990). On the large deviation principle by the asymptotic value method. In Pinsky, M. (Ed.), Diffusion Processes and Related Problems in Analysis, volume I, (pp. 447-472). Birkhäuser.

[4] Chan, T. (1993). Large deviations for empirical measures with degenerate limiting distribution. Probab. Theory Related Fields, 97(1-2), 179-193.

[5] de Acosta, A. (1985). Upper bounds for large deviations of dependent random vectors. $Z$. Wahrsch. Verw. Gebiete, 69(4), 551-565.

[6] Dembo, A. \& Zeitouni, O. (1998). Large deviations techniques and applications (Second ed.). New York: Springer-Verlag.

[7] Dinwoodie, I. H. \& Zabell, S. L. (1992). Large deviations for exchangeable random vectors. Ann. Probab., 20(3), 1147-1166.

[8] Hiai, F. \& Petz, D. (2000). The semicircle law, free random variables and entropy. Providence, RI: American Mathematical Society.

[9] Johansson, K. (1998). On fluctuations of eigenvalues of random Hermitian matrices. Duke Math. J., 91(1), 151-204.

[10] Ressel, P. (1982). A general Hoeffding type inequality. Z. Wahrsch. Verw. Gebiete, 61(2), $223-235$.

Department of Mathematics, University of Cincinnati, P.O. Box 210025, Cincinnati, OH 45221-0025, Whodzimierz.BryC@UC.EDU 